\title{
EP-79
}

\section{Renoportal anastomosis in adult-to-adult living donor liver transplantation for patients with portal vein thrombosis in single center experience}

\author{
Deok-Bog MOON* , Sung-Gyu LEE, Young-In YOON
}

Division of Hepatobiliary Surgery and Liver Transplantation, Asan Medical Center, University of Ulsan College of Medicine, Seoul, Korea

Introduction: The anastomosis between the left renal vein and graft portal vein is one of the crucial options in patients with end-stage liver disease with portal venous thrombosis and a previous splenorenal shunt. However, especially in living donor liver transplantation (LDLT) practice, limited cases are published in the literature.

Methods: Between January 2006 and December 2019, a total of 14 patients underwent adult LDLTs with renoportal anastomosis (RPA). The long-term outcomes were evaluated by reviewing the medical records of the 14 patients.

Results: Of the 14 patients who received adult LDLTs with RPA, 2 patients underwent end-to-end (E-to-E) RPA using left renal vein (LRV), 5 patients underwent side-to-end (S-to-E) RPA, and the rest underwent E-to-E RPA using LRV connected IVC cuff. Except two patients who died from cerebral hemorrhage and graft failure came from acute cellular rejection, twelve patients has maintained normal graft function and a patent RPA at a median follow-up of 42 months (range, 1-155 months).

Conclusions: RPA can be performed safely and effectively in LDLT as an acceptable and life-saving procedure for patients with endstage liver disease with portal venous thrombosis and a previous splenorenal shunt. According to our experience, E-to-E RPA using LRV connected IVC cuff is the best option considering feasibility and safety. 